\title{
Dexterous Machining Aiming at High Value-added Products
}

\author{
Yoshimi Takeuchi'"\#
}

1 Department of Mechanical Engineering, Chubu University, Matsumoto-cho 1200, Kasugai, Aichi, Japan, 487-8501 \# Corresponding Author / E-mail: takeuchi_yoshimi@isc.chubu.ac.jp, TEL: +81-568-51-9666, FAX: +81-568-51-1194

KEYWORDS: Dexterous machining, Value-added product, Multi-tasking machine tool, Artistic product

\begin{abstract}
It is important to establish a new approach of creating such value-added products as craftworks or applied arts, different from traditional way of achieving products with high quality and low price. Thus, the study deals with the first trial of creating artistic product shapes by means of multi-axis controlled multi-tasking machine tools or multi-axis controlled machining centers on the basis of mechanical cutting technology. The concept of creating high value-added products by devising cutting ways is named as "Dexterous Machining". In the study, "Dexterous Machining" is classified into several areas and demonstrated. As a result, it is experimentally found that the concept of "Dexterous Machining" has the potential of creating high value-added products.
\end{abstract}

Manuscript received: May 4, 2014 / Revised: May 12, 2014 / Accepted: May 12, 2014 (Invited Paper)

\section{Introduction}

In recent years, complicated shapes to be created in a short time have being increased. Machine tools have become highly accurate and had the multifunction of turning and milling as well as the enhancement of CAM systems. However, there exist a lot of complicated shapes difficult or impossible to machine even by means of multi-axis control machine tools. Most of them are made by the ingenuity of skilled persons. In the near future, it is predicted that consumer needs are getting more various and that consumers require individual goods matching for their feeling.

Thinking the modern manufacturing technology, a lot of complicated product shapes are firstly divided into simple parts and machined to satisfy the compatibility with other parts, taking account of dimension accuracy, form accuracy and surface roughness. Then, they are assembled as final products. On the other hand, the creation of complicated shapes like artistic products without dividing into simple parts can lead to products with added value. Such a machining technology may be called "Dexterous Machining".

"Dexterous Machining" may be classified into several areas; (1) extremely complex shape creation, (2) machining of workpieces with extremely unstable shape, (3) machining of nested shapes, and (4) machining of soft materials difficult to fix.

The study demonstrates the results of "Dexterous Machining" with regard to the above four areas, which are created by use of a 5 -axis controlled multi- tasking machine tool and a 5-axis controlled machining center. Fig. 1 shows a multi-tasking machine tool to achieve turning and milling operations in one clumping or chucking, which consists of $\mathrm{X}, \mathrm{Y}$ and $\mathrm{Z}$ - axis as the translation axes, and $\mathrm{B}$ and $\mathrm{C}$-axis as the rotational axes. In addition, it has $\mathrm{A}$-axis as another translation axis and $\mathrm{C} 2$-axis as a rotational axis on the second spindle so that a workpiece can be passed from the first one to second one, or is separated with grasping both side of the workpiece. The proposed "Dexterous Machining reflects these features of a multi-tasking machine tool.

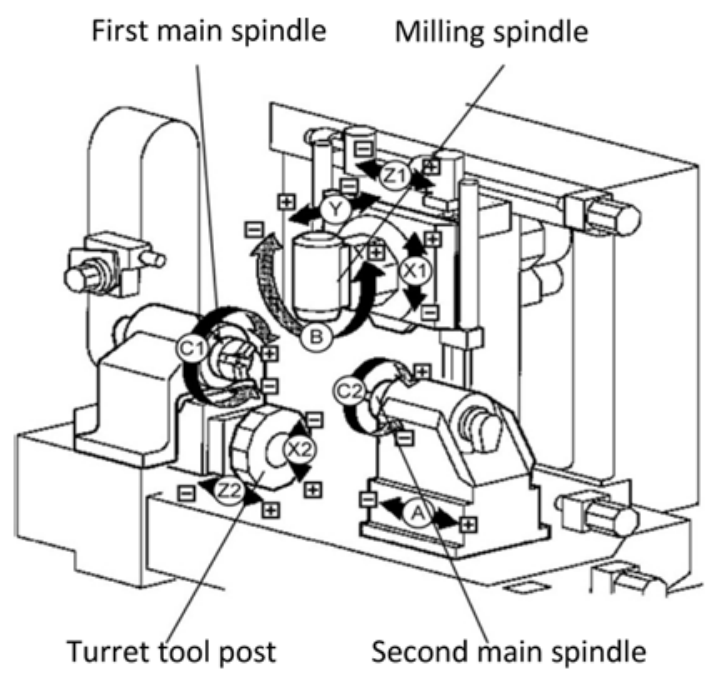

Fig. 1 Structure of 5-axis controlled multi-tasking machine tool 


\section{Extremely Complex Shape Creation}

\subsection{Chain-shaped Ring}

As an example of artistic product shapes, a chain-shaped ring shown in Fig. 2 is selected in this study. ${ }^{1}$ Though there are various CAM systems for multi-tasking machine tools, it is difficult to deal with this kind of shape since the interference occurs among a cutting tool and workpieces. Therefore, a special CAM system is developed by taking account of these interferences. In order to machine the chain-shaped ring, the following three stages are required; rough cutting before separation, separation of the workpiece into several parts and finish cutting. In rough cutting and separation, it is important to consider the shape just before separation and to devise how to divide the workpiece. In addition, the interference avoidance among a cutting tool and a workpiece, especially between divided parts, should be considered in finish cutting.

Then, the bridges connecting two parts are generated in rough cutting. A bridge is prepared for two overlapped parts when they are projected to a certain plane. The bridge is added to link the whole parts since the chain-shaped ring is machined as one workpiece in rough cutting. Finally, the shape is machined, while remaining bridges shown in Fig. 3.

Fig. 4 shows the simulation screen (NC simulator: G-Navi) to check the NC codes generated in the system. These simulation results show that the generated rough cutting NC codes successfully machine the targeted chain-shaped ring without any inappropriate interference.

After rough cutting, the bridges are removed from the workpiece by using a jig, as shown in Fig. 5(a). Also, the finished chain-shapes are separated from the both ends of the workpiece, as shown in Fig. 5(b).

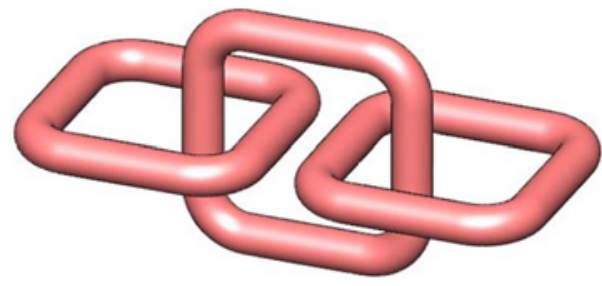

Fig. 2 Chain-shaped ring

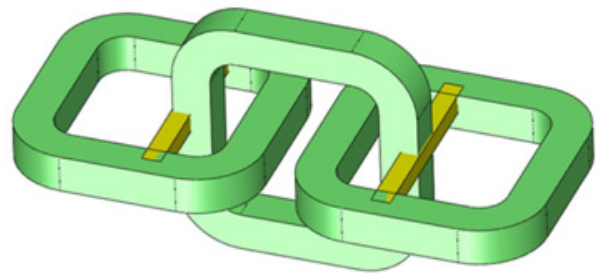

Fig. 3 Rough cut rings connected by bridges

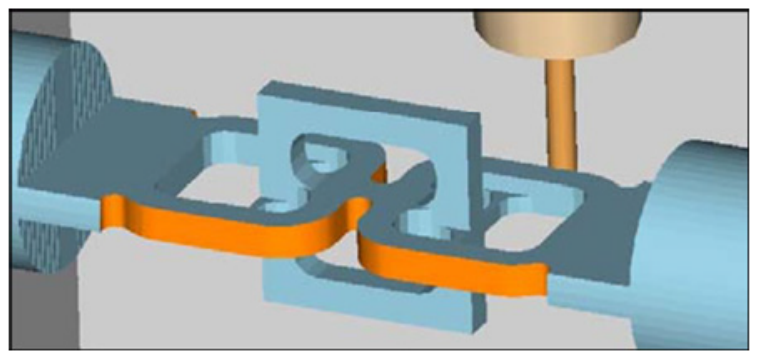

Fig. 4 Simulation of rough cutting
Separated parts are supported by the jig to continue the following finish cutting.

Photographs of the workpiece during and after machining are shown in Fig. 6. It is found that the machining experiments are adequately completed to create the chain-shaped ring defined by 3D-CAD models.

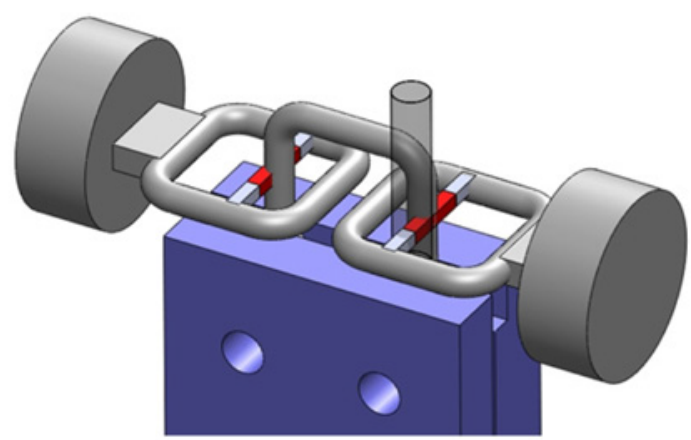

(a) Clumping of the middle ring to remove bridges

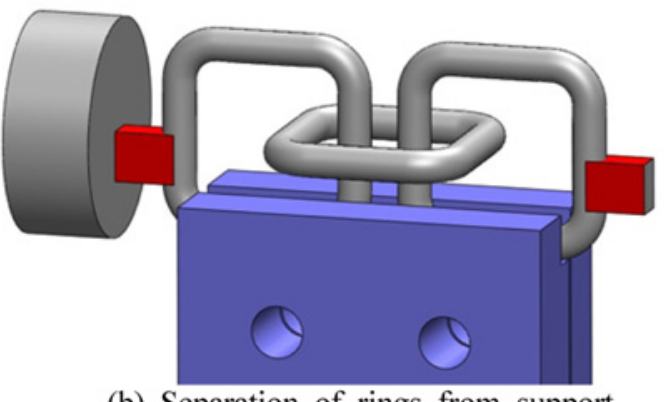

(b) Separation of rings from support

Fig. 5 Bridge removal and separation of rings

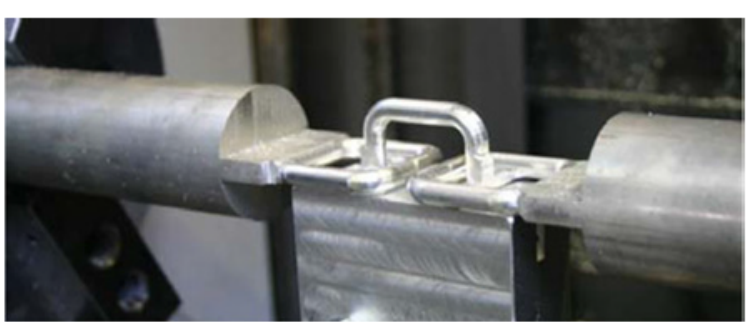

(a) Clamping of the middle ring

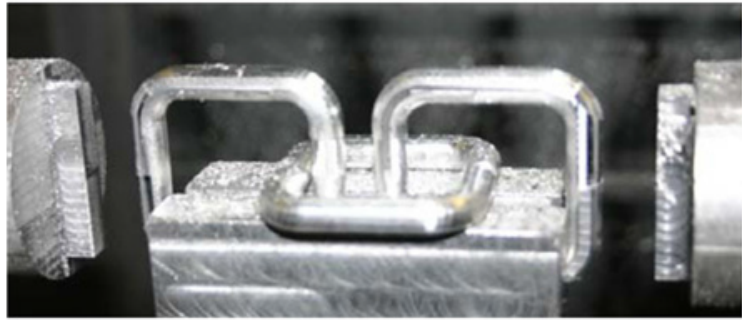

(b) Separation of rings from support

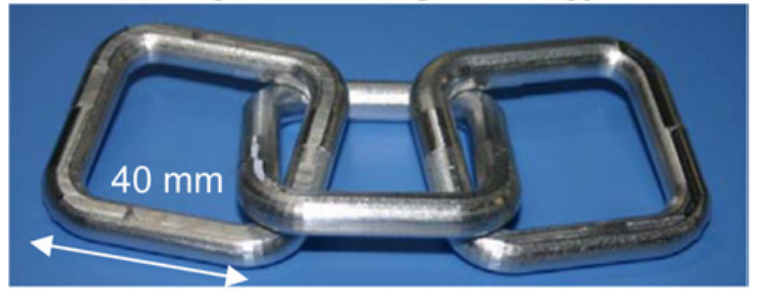

(c) Finished chain-shaped ring

Fig. 6 Creation of chain-shaped rings from bar material 


\subsection{Bended Columns}

The shape of bended columns consists of three or four curved cylinders between two disks, ${ }^{2}$ as illustrated in Fig. 7. When the bended column is machined, the tool is moved along the longitudinal direction of the bended column. In rough cutting, turning and turn-milling are applied to make the target shape. For the purpose, the loft-surface is created between the upper disk and the lower disk. At the time, the surface of bended columns is offset, taking account of the radius of ball end mill for finish cutting. Then, the developed CAM system generates 5 -axis control tool paths for rough cutting and finish cutting, taking the interference avoidance into account. Finally, these tool paths are output as CL data, which are then transformed into NC data by the post processer.

By using two shapes shown in Fig. 7, the simulations are conducted respectively, as shown in Fig. 8.

Then, actual cutting experiments are made by use of a square end mill for rough cutting and a ball end mill for finish cutting. As a result, the bended columns are well created, as seen in Fig. 9.

\section{Machining of Workpieces with Extremely Unstable Shape}

Unstable shape means a body with a high aspect ratio and large mass at the top of the body. Such a shape is difficult to machine due to its low stiffness. Cutting force causes the breakage of a body. Fig. 10 shows such a shape, which has a long column with a large mass. The system judges the possibility of machining without breakage by use of FEM. If the breakage is judged to take place, the system adds several support pillars so that the milling tool cannot break the body during operation. The number of support
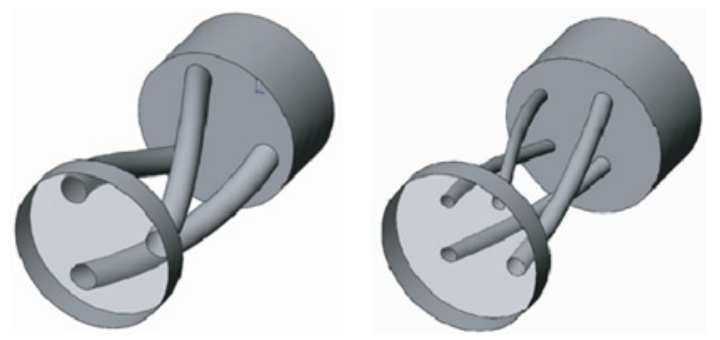

Fig. 7 Example of bended columns
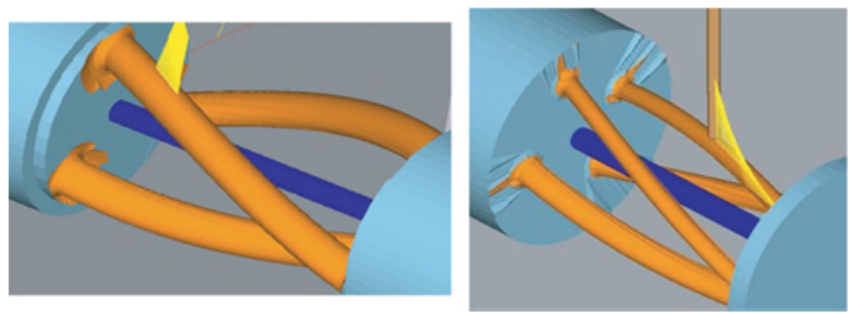

Fig. 8 Simulation of generated tool paths
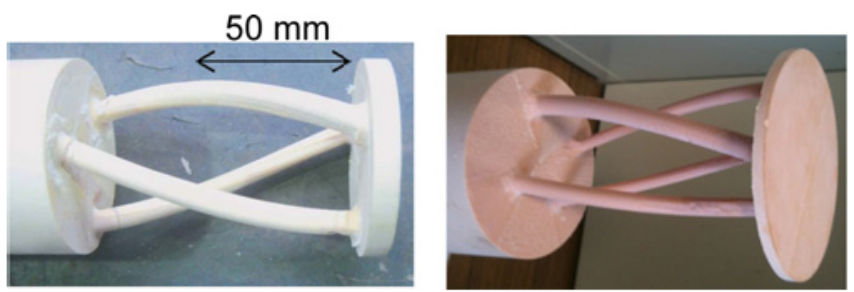

Fig. 9 Machined results pillars is determined by reference to the calculated result of FEM.

Fig. 11(a) shows the simulated results of machining the unstable shape, where three support pillars are added to the body. After finishing the machining, taking account of the interference between the cutting tool and the body, the support pillars are removed, as shown in Fig. 11(b). Based on the tool paths for rough cutting and finish cutting, the cutting experiment is carried out and the machined body is obtained, as shown in Fig. 12(a) with support pillars and in Fig. 12(b) without support pillars. From this figure, it is seen that the system can create the unstable shape same as the defined one.

\section{Creation of Nested Shape}

A nested shape is defined to be a body (an outer part) having an inner part in it. Let's show a typical nested shape, as illustrated in Fig. 13,

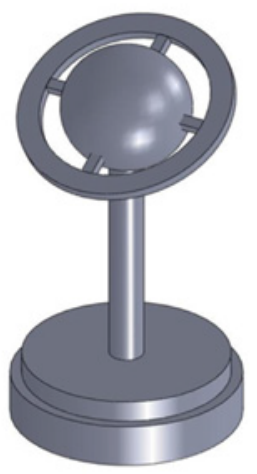

Fig. 10 Example of unstable shape

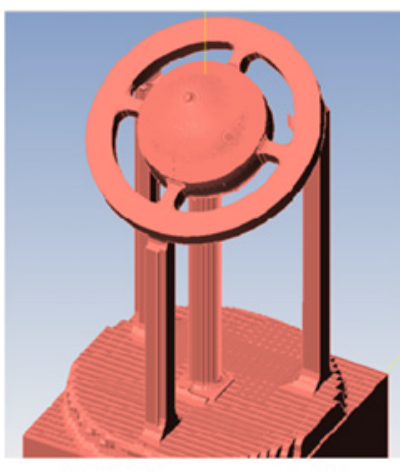

(a) With support pillar

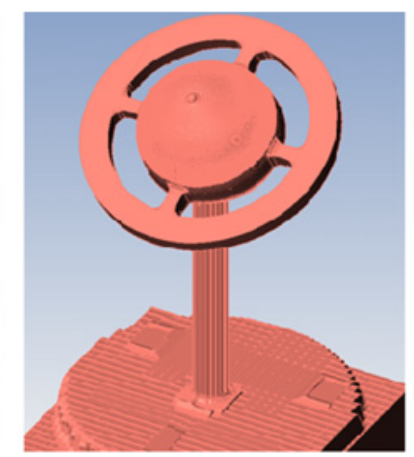

(b) Removal of support pillar
Fig. 11 Simulated result of machining

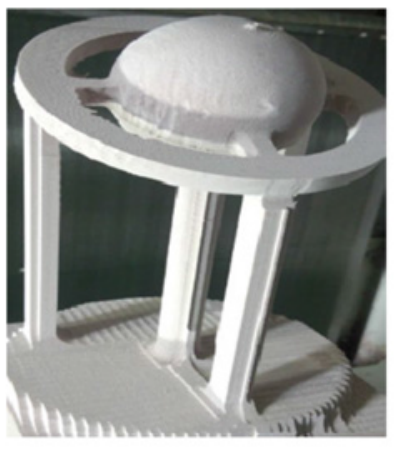

(a) With support pillar

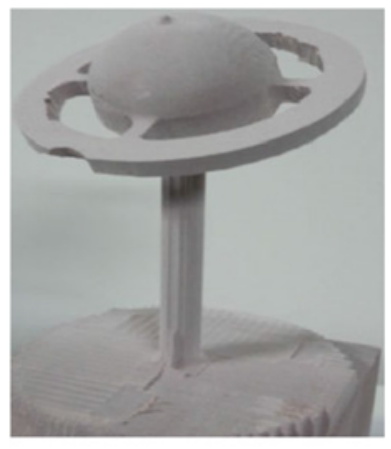

(b) Removal of support pillar
Fig. 12 Machined result of unstable shape 
consisting of a ball as an inner part in a cube with six small holes as an outer part. $^{3}$

Fig. 14 shows the procedure of dexterous machining of the nested shape. At first, a cylindrical workpiece as a blank to the multi-tasking machine is chucked by the first main spindle and machined to be the same shape as the outer part of the targeted shape, as shown in Fig. 14(a). Furthermore, five holes are made on the faces except for the face against the first main spindle. Then, the inner part to become a ball is machined as "Support Pillar" in the side of the first spindle.

The inner part and an outer part are separated by a grooving tool, while rotating the workpiece around its center-axis corresponding to zaxis, as shown in Fig. 14(b). During the process, the outer part must be fixed by a jig. The jig is chucked by the second main spindle. After separation, finish cutting is performed for the inside of the outer part. To rotate the inner part around $\mathrm{C}$-axis, tool paths are generated on $\mathrm{X}$ $\mathrm{Y}$ plane. In the case of a ball as the inner part, tool paths are generated, as shown in Fig. 14(c). A cutting tool can machine a large area by rotating the inner part by $360 \mathrm{deg}$. in the direction of C-axis at each tool posture. The movement of the support pillar by the first main spindle and the outer part by the second main spindle in A-axis direction can enlarge a machining area from one hole, as shown in Fig. 15.

Finally, the support pillar is cut down, and the remained part is removed, as shown in Fig. 14(d). At this time, the ball as an inner part is fixed by a jig to be machined. The CAM system generates NC data for both rough cutting and finish cutting to form the ball. The CAM system makes the most of API functions of "Solid Works".

Based on the above procedure to create a nested shape, the cutting experiment is carried out. The targeted shape consists of a cube (each
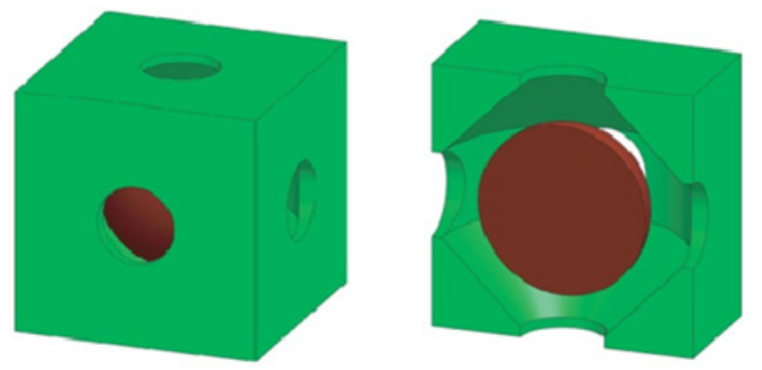

Fig. 13 Nested shape consisting of cube and ball

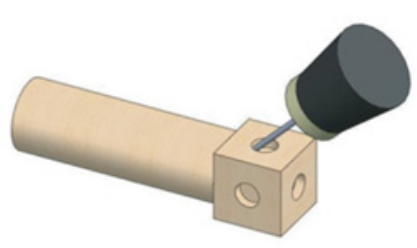

(a) Rough cutting of nested shape

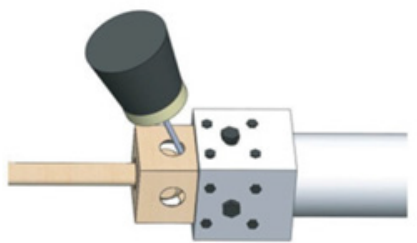

(c) Finish cutting of inner part

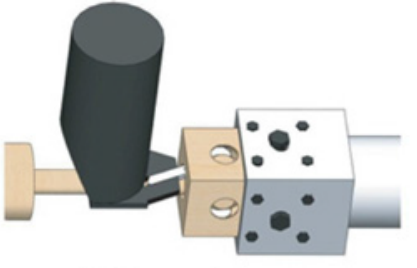

(b) Separation
Fig. 14 Procedure of creating nested shape edge length is $50 \mathrm{~mm}$ ) with six holes of $18 \mathrm{~mm}$ in diameter as an outer part and a ball of $32 \mathrm{~mm}$ in diameter as an inner part. Square end mill of $6 \mathrm{~mm}$ in diameter and a ball end mill of $2 \mathrm{~mm}$ in radius are used in the experiment. The support pillar is generated without any problems, as shown in Fig. 16, and it is found that the same shape as the targeted shape is obtained, as shown in Fig. 17.

\section{Machining of Soft Materials Difficult to Fix and Grasp}

The study deals with the first trial of creating an artistic product shape of soft objects. For the making of soft objects such as rubber, the mold is usually employed. However, mold fabrication is not suitable for small lot production. On the other hand, it is difficult to fix soft objects on the table. The cryogenic machining of a viscoelastic polymer was proposed to fix them, it is expensive to make common products and difficult to control the temperature, while machining. ${ }^{4}$

To overcome this problem, the study proposes a new method to clamp soft material and to suppress its deformation by employing an aqueous solution of sodium acetate. Let's show an example of creating a soft object with thin wall, which cannot usually be machined in the conventional manner. ${ }^{5}$ As a soft material, urethane rubber is selected, which has very low stiffness and is difficult to precisely machine because of the deformation

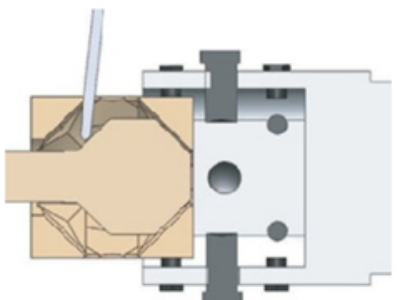

(a) Machining the left side

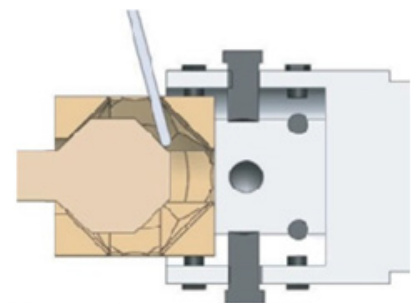

(b) Machining the right side
Fig. 15 Machining of the inner part with jig

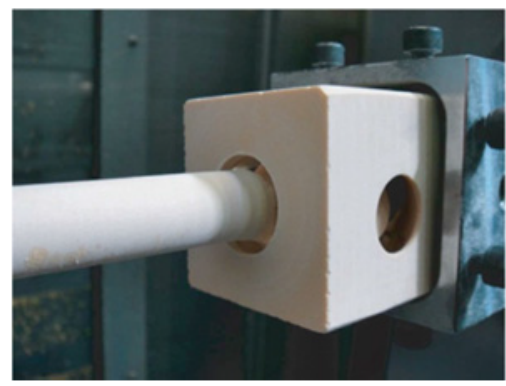

Fig. 16 Machining of support pillar

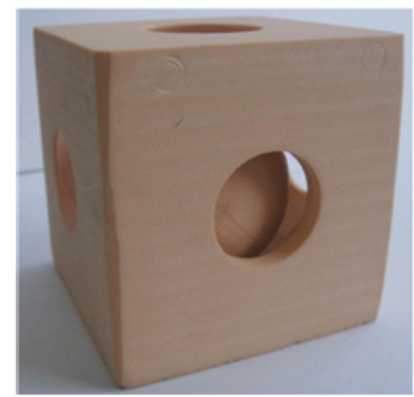

Fig. 17 Machined shape 
caused by not only the cutting force but also clamping force itself.

Aqueous solution of sodium acetate, known as hot ice, is used as a flexible clamping device. Sodium acetate trihydrate crystals melt at about $330 \mathrm{~K}$ dissolving in their water of crystallization. When they are heated past the melting point, the aqueous solution becomes supersaturated. By applying stimulus in the solution, a nucleation core is formed which causes the solution to crystallize into solid sodium acetate trihydrate again. ${ }^{6,7}$ Since the phenomena depend on reversible chemical reactions, sodium acetate heat packs can be easily recharged by placing in boiling water and be reused many times.

Fig. 18 shows our proposed soft object machining by means of flexible clamper. Soft object is located in the case filled by an aqueous solution of sodium acetate on a horizontal machining center table. Then, the aqueous solution is crystallized by applying stimulus, and the solid sodium acetate clamps the soft object. Because the soft object is surrounded by the aqueous solution, even complicated shape can be uniformly fixed and suppress the local clamping deformation.

The solid sodium acetate, as flexible clamper, is simultaneously removed while machining. It is possible to recharge the aqueous solution into the removed part for increasing the apparent stiffness, as illustrated in Fig. 19. As a soft object, wine glass with thin wall is created.

Fig. 20 shows a machined wine glass made of urethane rubber. As a result, it is experimentally found that the usage of the aqueous solution of sodium acetate has the potential of realizing "Dexterous Machining of soft objects like urethane rubber.

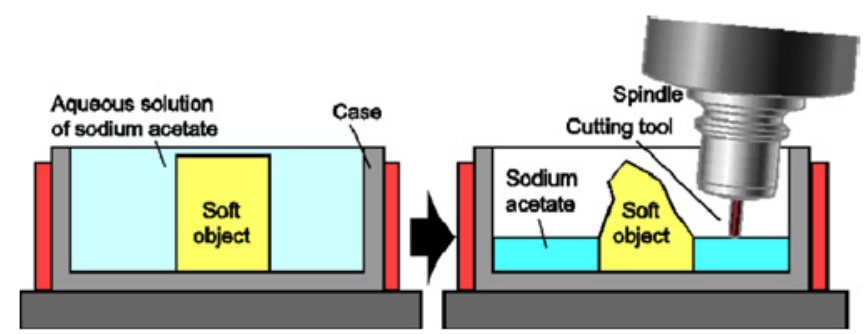

Fig. 18 Fixing method of soft object by aqueous solution of sodium acetate

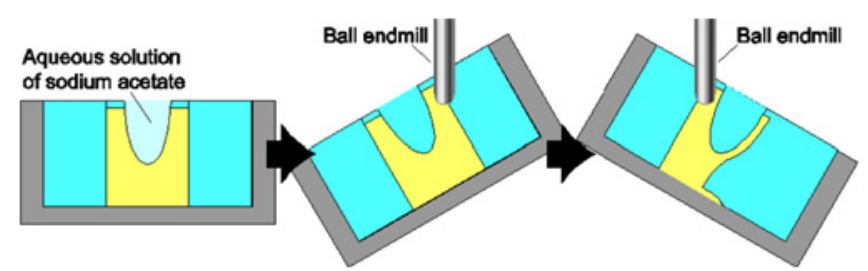

Fig. 19 Recharge of sodium acetate decreasing with cutting
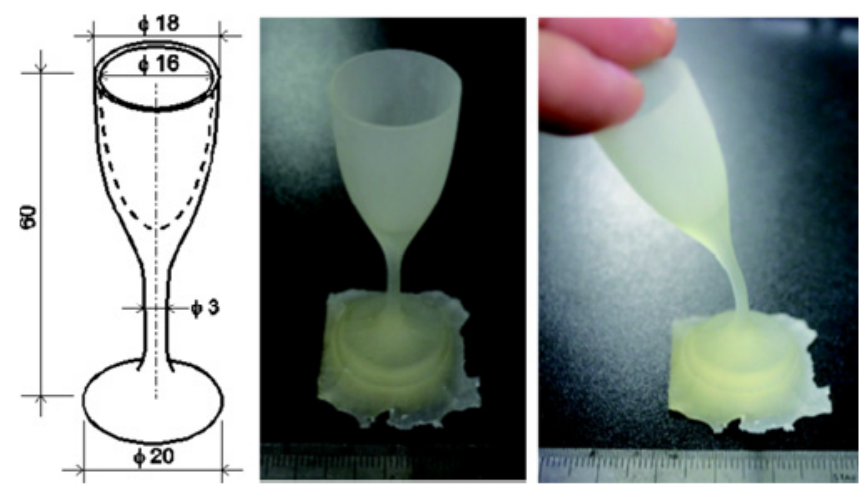

Fig. 20 Machined wine glass shape of urethane

\section{Conclusions}

The study proposes a new concept of "Dexterous Machining" to create high value-added products. "Dexterous Machining" can be classified into several areas. In the study, four typical areas are introduced with machined examples. As a result, it is seen that "Dexterous Machining" has the potential of creating high value-added products.

\section{ACKNOWLEDGEMENT}

The study is partially supported by Grant-in-Aid for Scientific Research (No. 23360069) of the Japan Society for the Promotion of Science (JSPS). In addition, the author would like to express sincere thanks to DMG MORI SEIKI Co., DT Technology, YAMAZAKI MAZAK Co., Aikoku Alpha Co. and OSG Co. for their cooperation, to Dr. K. Nakamoto for his collaboration and to our students, R. Okamine, Y. Ieda and S. Yamamoto, for their earnest assistance to experiments.

\section{REFERENCES}

1. Nakamoto, K., Fujimoto, T., Kawaguchi, Y., Ishida, T., and Takeuchi, Y., "Dexterous Machining of Chain-shapes by Employing a Multi-tasking Machine Tool," Proc. of the $15^{\text {th }}$ Intemational Conference on Mechatronics Technology in Melbourne, pp. 59-62, 2011.

2. Natsume, N., Nakamoto, K., Ishida, T., and Takeuchi, Y., "Dexterous Machining of Complicated Shape Consisting of Bended Columns," Proc. of the $14^{\text {th }}$ International Conference on Mechatronics Technology in Osaka, pp. 24-27, 2010.

3. Hamada, D., Nakamoto, K., Ishida, T., and Takeuchi, Y., "Dexterous Machining for a Complicated Shape with Nested Structure," Proc. of the $14^{\text {th }}$ International Conference on Mechatronics Technology in Osaka, pp. 28-31, 2010.

4. Kakinuma, Y., Kidani, S., and Aoyama, T., "Ultra-precision Cryogenic Machining of Viscoelastic Polymers," CIRP AnnalsManufacturing Technology, Vol. 61, No. 1, pp. 79-82, 2012.

5. Nakamoto, K., Ueji, R., and Takeuchi, Y., "Dexterous Machining of Soft Objects Difficult to Clump," Transactions of the Japan Society of Mechanical Engineers Series C, Vol. 79, No. 808, pp. 4535-4542, 2013.

6. Cabeza, L. F., Svensson, G., Hiebler, S., and Mehling, H., "Thermal Performance of Sodium Acetate Trihydrate Thickened with Different Materials as Phase Change Energy Storage Material," Applied Thermal Engineering, Vol. 23, No. 13, pp. 1697-1704, 2003.

7. Furbo, S., Fan, J., Andersen, E., Chen, Z., Chen, Z., et al., "Development of Seasonal Heat Storage based on Stable Supercooling of a Sodium Acetate Water Mixture," Energy Procedia, Vol. 30, pp. 260-269, 2012. 